\title{
Fungal Translocation Is Associated with Immune Activation and Systemic Inflammation in Treated HIV
}

\author{
Lukasz D. Weiner, ${ }^{1,2}$ Mauricio Retuerto, ${ }^{3,4}$ Christopher L. Hager, ${ }^{3,4}$ Vanessa El Kamari, ${ }^{3}$ \\ Lingpeng Shan, ${ }^{3}$ Abdus Sattar, ${ }^{3}$ Manjusha Kulkarni, ${ }^{5}$ Nicholas Funderburg, ${ }^{5}$ Mahmoud A. Ghannoum, ${ }^{3,4}$ \\ Sahera Dirajlal-Fargo, ${ }^{1-3}$ and Grace A. McComsey ${ }^{1-3}$
}

\begin{abstract}
The mechanisms causing HIV-associated immune activation remain incompletely understood. Alteration of intestinal integrity with subsequent translocation of bacterial products appears to play an important role; however, little is known about the impact of fungal translocation. We assessed the effect of fungal translocation and its association with immune activation in people living with HIV (PLWH) compared with uninfected controls. We measured serum levels of $\beta$-D-glucan (BDG) and anti-Saccharomyces cerevisiae antibodies (ASCA) immunoglobulin $\mathrm{G}(\operatorname{IgG})$ and immunoglobulin $\mathrm{A}(\operatorname{IgA})$ and markers of systemic inflammation and immune activation in virally suppressed PLWH on antiretroviral therapy (ART) and uninfected controls. T-test and Mann-Whitney tests were used to compare markers by HIV status and correlation and regression analyses were used to assess associations of fungal translocation markers with markers of inflammation. One hundred seventysix participants were included (128 HIV+ and $48 \mathrm{HIV}-$ ); $72 \%$ male, 65\% African American, median age was 50 years, and CD4 was 710 cells $/ \mathrm{cm}^{3}$. Levels of BDG tended to be lower in HIV+ when compared with controls $(p=.05)$. No significant difference in levels of ASCA IgG and IgA was seen between groups $(p>.75)$. There was a significant correlation between BDG and several markers of inflammation and immune activation in PLWH, not seen in uninfected controls. In contrast, no correlations were seen between levels of ASCA IgG and IgA with inflammatory markers. PLWH on ART do not have higher levels of BDG or ASCA when compared with uninfected controls, however, the association found between BDG and several inflammation markers suggests a potential role of fungal translocation in the heightened immune activation seen in treated HIV.
\end{abstract}

Keywords: HIV, fungi, inflammation, gastrointestinal microbiome, biomarkers

\section{Introduction}

D ESPITE VIROLOGIC CONTROL on antiretroviral therapy (ART), people living with HIV (PLWH) continue to have overall increased mortality and decreased life expectancy when compared with the general population. ${ }^{1-3}$ Heightened systemic inflammation, as demonstrated by activation of both cellular and innate immunities, remains persistently elevated in PLWH despite viral suppression. ${ }^{4,5}$ This persistent inflammation has been correlated to subclinical cardiovascular disease (CVD) and an increase in the prevalence of age-associated noncommunicable comorbidities among PLWH. ${ }^{6-8}$ The etiology of this heightened in- flammation is likely multifactorial and remains incompletely understood, but the role of alteration of intestinal integrity and the resultant translocation of bacterial products through the intestinal lumen to the systemic circulation appears to be a central factor. ${ }^{9,10}$

Compromise of the integrity of the intestinal mucosa secondary to HIV infection ${ }^{11,12}$ allows for translocation of bacterial products such as lipopolysaccharide (LPS), which stimulates the downstream transcription and activation of proinflammatory mediators. ${ }^{13}$ Although fungi are an integral part of the human microbiota, their contribution to systemic inflammation remains largely unknown. Markers such as $\beta$-D-glucan (BDG) and anti-Saccharomyces cerevisiae

\footnotetext{
${ }^{1}$ Pediatric Infectious Diseases, University Hospitals Cleveland Medical Center, Cleveland, Ohio.

${ }^{2}$ Rainbow Babies and Children's Hospital, Cleveland, Ohio.

${ }^{3}$ Case Western Reserve University, Cleveland, Ohio.

${ }^{4}$ Center for Medical Mycology, Case Western Reserve University and University Hospitals Cleveland Medical Center, Cleveland, Ohio.

${ }^{5}$ Ohio State University School of Health and Rehabilitation Sciences, Columbus, Ohio.
} 
antibodies (ASCA) have been demonstrated in the serum of individuals with invasive fungal disease or in those with compromised intestinal integrity, such as occurring with inflammatory bowel disease (IBD). ${ }^{14,15}$ Through interaction with receptors belonging to the C-type lectin superfamily, fungal markers such as BDG are known activators of proinflammatory cascades. ${ }^{16,17}$

Our objective was to compare the fungal translocation markers BDG and ASCA immunoglobulin G (IgG) and immunoglobulin A (IgA) in treated PLWH versus uninfected controls and study their relationship with systemic inflammation and cellular and innate immune activation.

\section{Materials and Methods}

\section{Study design and population}

This is a cross-sectional analysis using a cohort of PLWH and uninfected healthy participants of similar age, both cohorts prospectively enrolled at University Hospitals Cleveland Medical Center, Cleveland, Ohio. PLWH were included as part of a prospective cross-sectional assessment and were seen during their routine HIV care over a 2-year period. Healthy controls were recruited as volunteer University Hospital employees and/or family members of PLWH. The study was approved by the local institutional review board and written informed consent was obtained from each participant.

In both groups, participants were at least 18 years of age without a known history of CVD or other pre-existing inflammatory conditions. All PLWH had an HIV-1 RNA viral load less than 1,000 copies/mL in the past 4 months, were on stable ART for at least 3 months, and had cumulative ART use of at least 6 months. Participants in both groups were excluded if they had active bacterial or fungal infection, were pregnant or lactating, or on antifungal agents.

\section{Study evaluation}

Medical history and self-reported demographics were obtained at the initial screening visit as well as anthropometric data. Blood samples were obtained following a fasting period of at least $8 \mathrm{~h}$ and samples were prepared as previously described, ${ }^{18}$ with plasma and serum cryopreserved for measurement of markers of monocyte and cellular activation, systemic inflammation, and coagulation. The homeostatic model assessment of insulin resistance (HOMA-IR) was calculated. ${ }^{19}$ HIV parameters, including RNA levels and $\mathrm{CD}^{+}$cell counts, were obtained through routine care.

\section{Biomarkers of inflammation, immune activation, and fungal translocation}

Soluble markers of inflammation interleukin-6 (IL-6), soluble vascular cell adhesion molecule-1 (sVCAM-1), markers of immune activation (sCD14, sCD163, CD $4^{+} \mathrm{CD} 38^{+} \mathrm{DR}^{+}$, $\mathrm{CD}^{+} \mathrm{CD} 38^{+} \mathrm{DR}^{+}$), soluble tumor necrosis factor- $\alpha$ receptors I and II (sTNFR-I and sTNFR-II, respectively), interferon- $\gamma$ inducible protein 10 (IP-10), high-sensitivity C-reactive protein (hs-CRP), and D-dimer were determined using an enzymelinked immunosorbent assay (ELISA) (R\&D Systems; Minneapolis, MN). As a measure of oxidant stress, oxidized low-density lipoprotein (LDL) was determined via ELISA (Mercodia, Uppsala, Sweden) as well as measure- ments for the concentrations of lipoprotein-associated phospholipase A2 (Lp-PLA2) (PLAC test; diaDexus, South San Francisco, CA). Using cryopreserved serum, the markers of fungal translocation BDG and ASCA were measured by ELISA (BDG: MyBioSource, San Diego, CA; ASCA IgG and IgA: Orgentec Diagnostika GmbH, Mainz, Germany).

\section{Statistical analysis}

The main objectives of this study were to compare levels of BDG and ASCA in PLWH with uninfected controls, and to assess the relationship between these gut markers and markers of systemic inflammation and immune activation in PLWH and uninfected controls.

Demographics, clinical characteristics, fasting metabolic parameters, immune activation and inflammatory markers, measures of CVD risk, and serum gut markers were described and compared between the two study groups. The median and interquartile ranges were used to describe continuous variables, while categorical variables were reported as frequency and percentage.

Descriptive statistics, $t$-tests, and chi-square tests were used to compare baseline factors. Within and between groups, changes in BDG and ASCA were tested using Wilcoxon ranksum test. In both groups, relationships between fungal translocation markers and inflammation and immune activation markers were assessed using Spearman correlation analysis.

In a subgroup analysis, Spearman correlation was used to evaluate relationships between elevated levels of ASCA IgA with inflammation and immune activation markers in PLWH and healthy uninfected controls. Participants with levels greater than the median level of ASCA IgA $(3.08 \mathrm{U} / \mathrm{mL})$ were selected. Overall, 93 participants were included for this analysis: 69 PLWH and 24 from the uninfected group.

\section{Results}

\section{Baseline characteristics}

This analysis included 176 participants: 128 were PLWH and 48 were HIV-uninfected. Demographic information and baseline characteristics are described in Table 1. Overall, the median age was 50 years, $72 \%$ were males and $65 \%$ were African Americans. Among PLWH, the median $\mathrm{CD}^{+}$cell count was 710 cells $/ \mathrm{cm}^{3}$ and HIV viral load 20 copies $/ \mathrm{mL}$. While no participants were receiving any antifungal agents, four PLWH were on antibiotics during this study, including one on trimethoprim/sulfamethoxazole, two on isoniazid, and one on benzathine penicillin injections.

Two participants from each study group were taking probiotics. There were more males in the PLWH group $(p<.001)$. Waist-to-hip ratio and triglycerides were higher in PLWH $(p<.05)$, however, total cholesterol and LDL were higher in controls $(p<.05)$. The control group had higher levels of D-dimer $(p=0.05)$, whereas PLWH had higher levels of IP-10 ( $p<.001)$, sTNFR-II ( $p=.049)$, and T cell activation, as measured by $\mathrm{CD}^{+}$and $\mathrm{CD}^{+} \mathrm{T}$ cells expressing CD38 and human leukocyte antigen-DR isotype (HLA-DR) $(p<.001)$.

\section{Fungal translocation markers}

Serum levels of BDG tended to be lower in PLWH when compared with uninfected controls (Fig. $1 ; p=.05$ ), whereas 
Table 1. Comparison of Baseline Characteristics Between People Living with HiV and HIV-Uninfected Groups (Median Values [Interquartile Range])

\begin{tabular}{|c|c|c|c|}
\hline Variables & $P L W H, \mathrm{n}=128$ & HIV uninfected, $\mathrm{n}=48$ & $\mathrm{p}$ \\
\hline \multicolumn{4}{|l|}{ Demographics } \\
\hline Age (years) & $48.98(43.25-54.91)$ & $49.78(41.7-54.07)$ & .562 \\
\hline Male $(\%)$ & $105(82)$ & $23(48)$ & $<.001$ \\
\hline African American (\%) & $89(70)$ & $26(54)$ & .084 \\
\hline Family history of diabetes mellitus (\%) & $55(43)$ & $13(27)$ & .138 \\
\hline Family history of hypertension $(\%)$ & 87 (69) & $30(63)$ & .577 \\
\hline Current smoking $(\%)$ & $75(59)$ & $30(64)$ & .084 \\
\hline Alcohol $(\%)$ & $52(48)$ & $18(49)$ & .961 \\
\hline Current marijuana use $(\%)$ & $31(28)$ & $6(16)$ & .083 \\
\hline Current cocaine use $(\%)$ & $7(6)$ & $0(0)$ & .025 \\
\hline \multicolumn{4}{|l|}{ Cardiovascular disease risk factors } \\
\hline BMI $\left(\mathrm{kg} / \mathrm{m}^{2}\right)$ & $26.08(23.29-30.55)$ & $27.03(24.18-30.0)$ & .61 \\
\hline Waist-to-hip ratio & $0.96(0.9-1.01)$ & $0.91(0.85-0.97)$ & .004 \\
\hline Systolic blood pressure (mmHg) & $131.5(120.0-142.0)$ & $132.0(120.75-140.5)$ & .571 \\
\hline Diastolic blood pressure (mmHg) & $82.5(77.0-89.0)$ & $81.0(76.0-87.25)$ & .732 \\
\hline Framingham score & $4(2-8)$ & $3(1-7)$ & .078 \\
\hline Total cholesterol (mg/dL) & $168.0(143.75-193.0)$ & $186.0(161.5-213.25)$ & .004 \\
\hline HDL cholesterol (mg/dL) & $48.0(40.5-59.5)$ & $53.5(43.0-66.25)$ & .04 \\
\hline LDL cholesterol (mg/dL) & $88.5(71.0-108.0)$ & $106.0(82.75-131.25)$ & .002 \\
\hline Triglycerides $(\mathrm{mg} / \mathrm{dL})$ & $107.0(81.75-166)$ & $85.5(64.75-115)$ & .004 \\
\hline Pro-BNP $(\mathrm{pg} / \mathrm{mL})$ & $30(15.25-64)$ & $44.5(21.75-82.75)$ & .064 \\
\hline HOMA-IR & $2.46(1.41-4.28)$ & $2.09(1.63-3.14)$ & .365 \\
\hline \multicolumn{4}{|l|}{ Systemic markers of inflammation } \\
\hline $\mathrm{IP}-10(\mathrm{pg} / \mathrm{mL})$ & $141.67(84.95-235.46)$ & $58.77(34.26-87.16)$ & $<.001$ \\
\hline Lp-PLA (ng/mL) & $163(133-198.25)$ & $169.5(143-190.75)$ & .943 \\
\hline IL-6 $(\mu \mathrm{g} / \mathrm{mL})$ & $2.19(1.35-3.18)$ & $1.66(1.08-2.8)$ & .101 \\
\hline hs-CRP $(\mu \mathrm{g} / \mathrm{mL})$ & $1727.01(403.55-4376.33)$ & $1430.1(599.08-3535.84)$ & .995 \\
\hline Oxidized LDL (U/L) & $43.45(34.25-54.14)$ & $47.17(38.87-66.04)$ & .062 \\
\hline sTNFR-I (pg/mL) & 766.47 (536.91-1118.53) & $719.03(575.81-966)$ & .126 \\
\hline sTNFR-II (pg/mL) & $2482.08(2075.65-3224.77)$ & $2144.11(1908.8-2818.78)$ & .049 \\
\hline D-dimer $(\mu \mathrm{g} / \mathrm{mL})$ & $292.74(179.87-524.6)$ & $419.76(245.74-568.06)$ & .05 \\
\hline sVCAM-1 (pg/mL) & $762.35(627.44-928.01)$ & $753.68(594.98-920.18)$ & .55 \\
\hline \multicolumn{4}{|c|}{ Markers of innate and cellular immune activation } \\
\hline $\mathrm{sCD} 14(\mathrm{ng} / \mathrm{mL})$ & $1359(843-2010)$ & $1396(579-1920)$ & .337 \\
\hline $\mathrm{sCD} 163(\mathrm{ng} / \mathrm{mL})$ & $676.08(427.7-954.32)$ & $746.83(504.46-1221.05)$ & .183 \\
\hline $\mathrm{CD} 4^{+} \mathrm{CD} 38^{+} \mathrm{DR}^{+}(\%)$ & $4.54(3.21-6.29)$ & $3.11(2.3-3.96)$ & $<.001$ \\
\hline $\mathrm{CD}^{+} \mathrm{CD}^{+} 8^{+} \mathrm{DR}^{+}(\%)$ & $8.02(4.64-10.83)$ & $3.9(2.72-5.31)$ & $<.001$ \\
\hline \multicolumn{4}{|l|}{ HIV variables } \\
\hline Current CD4 count (cells $/ \mu \mathrm{L})$ & $710(537.75-932.75)$ & & \\
\hline Viral load (copies/mL) & $20(20-20)$ & & \\
\hline Nadir CD4 count (cells $/ \mu \mathrm{L})$ & $176(57.75-302.5)$ & & \\
\hline ART duration (months) & $113.31(64-168.61)$ & & \\
\hline \multicolumn{4}{|l|}{ Fungal markers of translocation } \\
\hline $\mathrm{BDG}(\mathrm{pg} / \mathrm{mL})$ & $149.22(119.78-200.76)$ & 166.13 (142.54-205.76) & .05 \\
\hline ASCA IgG (U/mL) & $5.97(2.88-13.28)$ & $6.02(4.33-11.39)$ & .747 \\
\hline ASCA IgA (U/mL) & $3.3(1.91-6.48)$ & $3.08(1.91-5.89)$ & .757 \\
\hline
\end{tabular}

ART, antiretroviral therapy; ASCA, anti-Saccharomyces cerevisiae antibodies; HDL, high-density lipoprotein; HOMA-IR, homeostatic model assessment of insulin resistance; hs-CRP, high-sensitivity C-reactive protein; IgA, immunoglobulin A; IgG, immunoglobulin G; IP10, interferon- $\gamma$-inducible protein 10; LDL, low-density lipoprotein; PLWH, people living with HIV; sTNFR, soluble tumor necrosis factor$\alpha$ receptors; sVCAM-1; soluble vascular cell adhesion molecule-1.

no difference was found in serum levels of ASCA IgG and IgA between the two groups (Fig. 2; $p=.75$, and Fig. 3; $p=.77$, respectively). No difference in serum levels of BDG or ASCA was observed between those with higher and lower CD4 cell counts categorized based on quartiles $(p \geq .45)$. Finally, no difference in serum BDG or ASCA was observed between male and female participants $(p \geq .10)$.

\section{Relationship between fungal translocation markers,} markers of systemic inflammation, and HIV variables

In univariable analyses, as shown in Figure 4, a weak but consistent correlation was observed between serum levels of BDG in PLWH and several inflammatory and monocyte activation markers, including sCD163, sCD14, IL-6, IP-10, sTNFR-II, D-dimer, and sVCAM-1. No correlation 


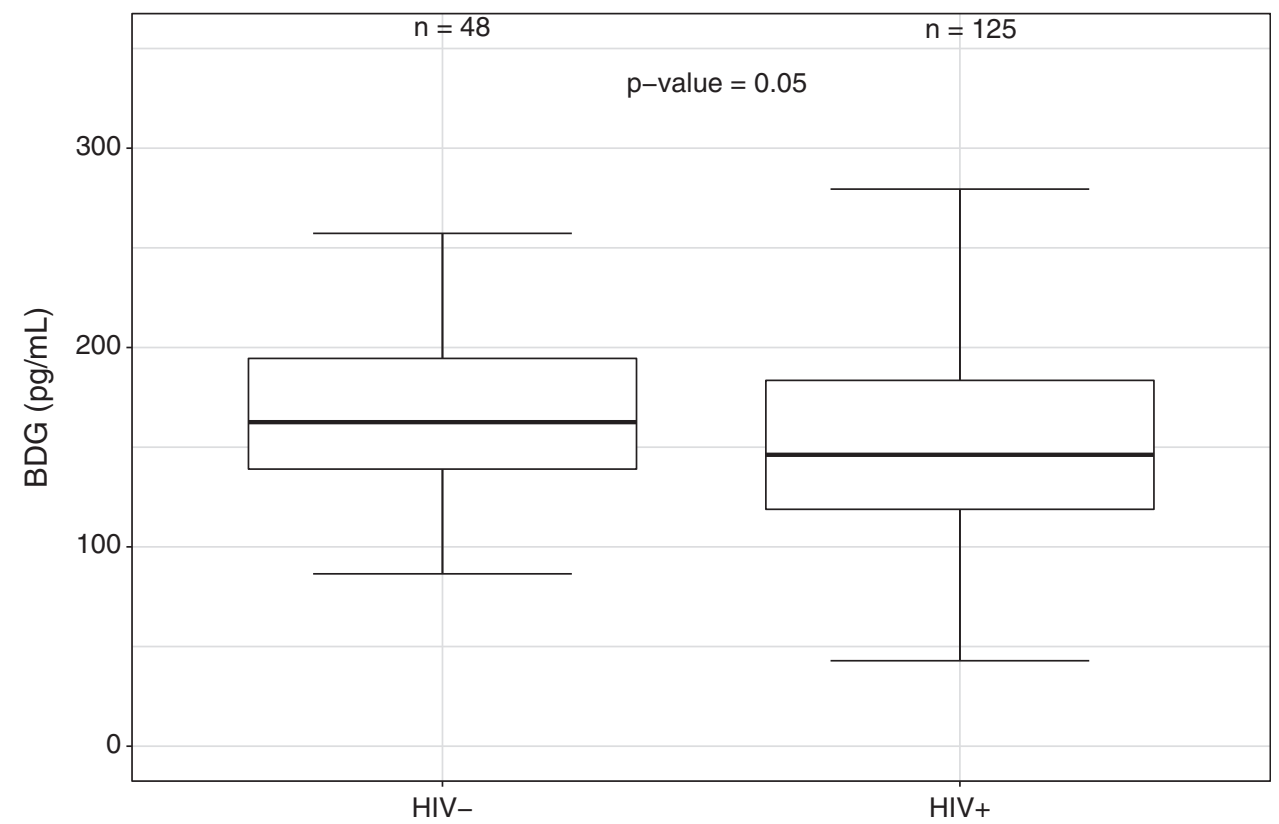

FIG. 1. Serum levels of $\beta$ D-glucan (BDG) in PLWH and in uninfected participants. PLWH, people living with human immunodeficiency virus. was found with Lp-PLA2, oxidized LDL, hs-CRP, sTNFR-I, and activated $\mathrm{CD}^{+}$and $\mathrm{CD}^{+} \mathrm{T}$ cells $(r=0.07-0.15 ; p=.08-$ .49). In addition, in these participants, only serum levels of sCD163 correlated with ASCA IgA (Fig. 5).

After adjusting for sex, IL6 and D-dimer no longer significantly correlated with BDG and SCD163 no longer correlated with ASCA $\lg A$

In uninfected participants, BDG was found to significantly correlate only with activated $\mathrm{CD}^{+}$and $\mathrm{CD}^{+} \mathrm{T}$ cells (Fig. 6). IP-10 significantly correlated with ASCA IgA (Fig. 7).

No correlation was observed between all HIV variables studied and fungal translocation markers. No correlation was seen between markers of fungal translocation and BMI or HOMA-IR $(p \geq .35)$.

\section{Relationship between elevated levels of ASCA $\lg A$ and markers of systemic inflammation and immune activation}

We found that a total of 93 participants had ASCA IgA levels greater than $3.08 \mathrm{U} / \mathrm{mL}$, the median value in uninfected participants, including 69 PLWH and 24 controls. As shown in Table 2, levels of ASCA IgA greater than $3.08 \mathrm{U} / \mathrm{mL}$ were found to be significantly associated with sCD14 in PLWH $(p=.012)$. In uninfected controls, higher levels of ASCA IgA significantly correlated with sTNFR-I and activated CD8+ T cells ( $p=.008$ and $p=.032$, respectively).

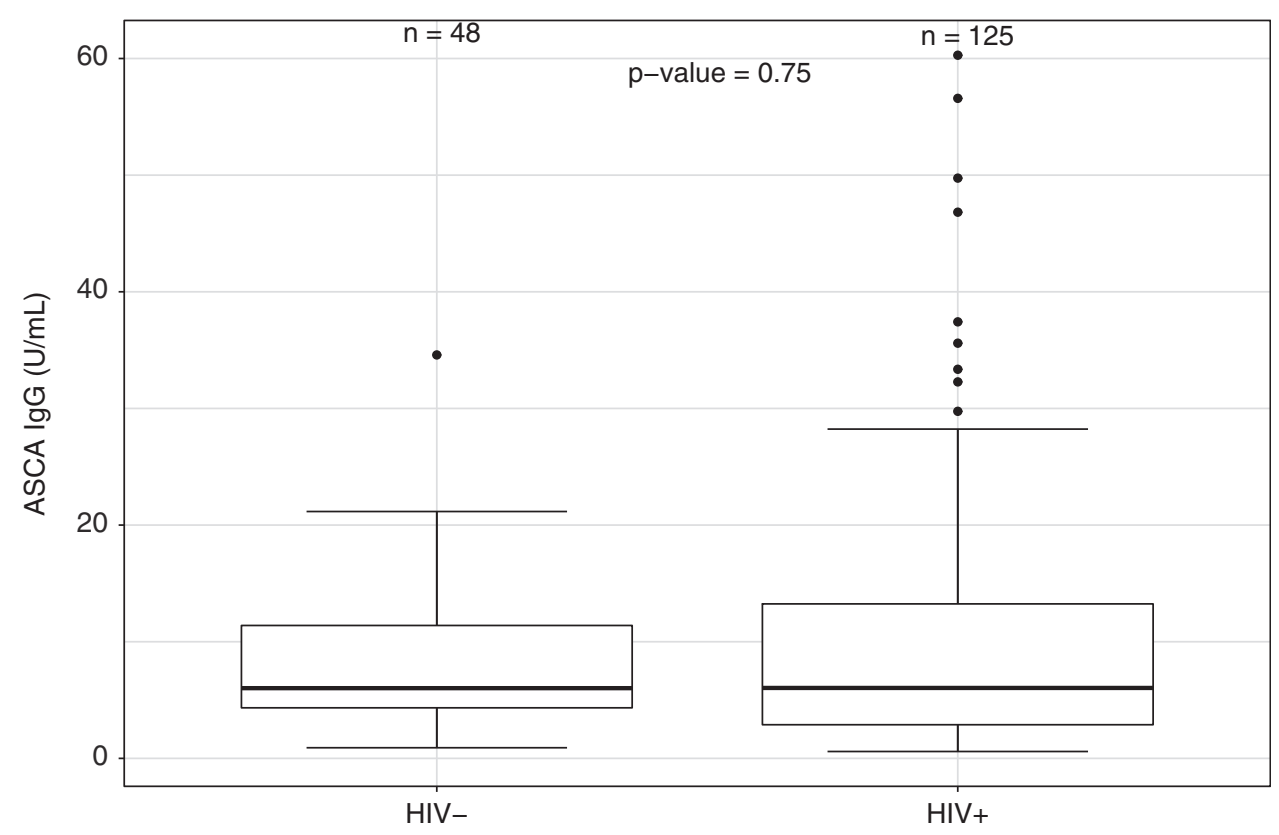

FIG. 2. Serum levels of ASCA IgG in PLWH and in uninfected participants. ASCA, anti-Saccharomyces cerevisiae antibodies. IgG, immunoglobulin G. 
FIG. 3. Serum levels of ASCA IgA in PLWH and in uninfected participants. IgA, immunoglobulin A.

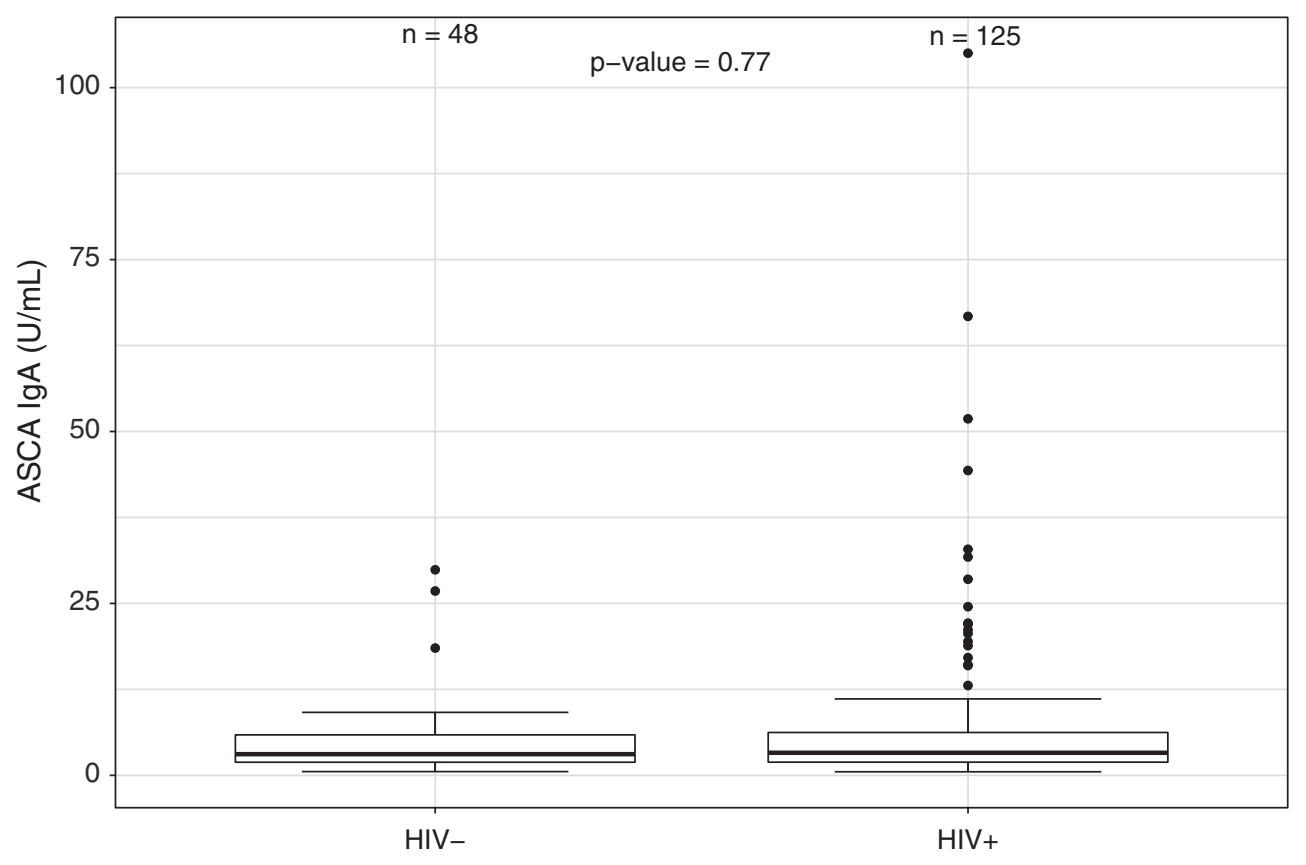

\section{Discussion}

To our knowledge, for the first time in PLWH, we investigated fungal translocation markers and their relationship with systemic inflammation and immune activation in comparison with uninfected controls. Our results suggest that overall, fungal translocation markers are not elevated in PLWH who are virally suppressed on ART when compared with levels in uninfected controls, however, this study does provide the first evidence that fungal translocation may be associated with the persistent inflammation and immune activation seen in treated HIV.

\section{Fungal translocation in HIV}

Most of the investigations in HIV describing the association between loss of gut integrity and systemic inflammation have focused on the measurement of LPS ${ }^{13,20}$ as a marker of bacterial translocation and a major contributor of the proinflammatory response. ${ }^{9,11,21-23}$ In addition, increased gastrointestinal (GI) permeability has been shown across multiple disease states, such as IBD and graft-versus-host-disease (GVHD), ${ }^{13,24}$ suggesting that increased gut permeability and translocation result in downstream activation of inflammation through receptor-mediated pathways.

Although fungal organisms contribute significantly to the composition of the normal GI flora, little is known regarding the translocation of fungal elements and their potential contribution to the systemic inflammatory response. The microbiome, referring specifically to the fungal component of microbiota, comprises $\sim 0.03 \%-2 \%$ of total gut microorganisms ${ }^{25}$ In inflammatory conditions outside of HIV, fungal translocation has been shown to affect human health, with systemic markers of fungal translocation being associated with disease activity and at times progression. For example, serum antibody against Saccharomyces cerevisiae (ASCA) has been used as a serologic marker for diagnosing Crohn's disease, with more recent investigations associating the detection of BDG in the serum with disease activity. ${ }^{26}$
Guo et al. demonstrated significantly higher levels of BDG in the serum of individuals with active Crohn's disease when compared with those in remission, and increased levels compared with those in healthy controls. ${ }^{27}$ In addition, serum levels of BDG in these participants correlated significantly with disease severity and elevated levels of erythrocyte sedimentation rate. Van der Valden et al. demonstrated an association between the rate of development of acute GVHD and fungal colonization (demonstrated as the presence of fungal species in fecal and/or mouthwash samples of participants) in poststem cell transplant patients. ${ }^{28}$ Finally, Kvehaugen $e t$ al. recently demonstrated a significant association between serum ASCA IgG and body fat mass and CRP in patients referred for morbid obesity, with elevated zonulin concentrations (a marker of intestinal permeability) being more frequently associated with ASCA IgG-positivity. ${ }^{29}$

Although a paucity of studies have investigated the role played by the microbiome in PLWH, to our knowledge there has been no study comparing markers of fungal translocation in PLWH versus uninfected healthy individuals.

We hypothesized that fungal translocation markers would be elevated in PLWH compared with levels in uninfected controls, which our findings do not support. Our findings could be either because there is truly no difference in these fungal markers between virally suppressed PLWH and uninfected controls, or our controls were not selected appropriately. It is also possible that the permeability of the GI tract in virally suppressed individuals is not sufficient to allow passage of fungal moieties in most individuals. Evidence of bacterial translocation persists despite antiretroviral treatment, ${ }^{9,21}$ but fungal markers such as ASCA and BDG less frequently reach the threshold of detection in non-HIV disease processes, such as Crohn's disease. ${ }^{26}$ The additional analysis and comparison of serum levels of LPS between our study groups may have been beneficial to demonstrate evidence of persistent gut translocation. The relationship between gut integrity and fungal moieties in HIV is likely complex and larger study sizes are needed to detect clinically significant effects. 

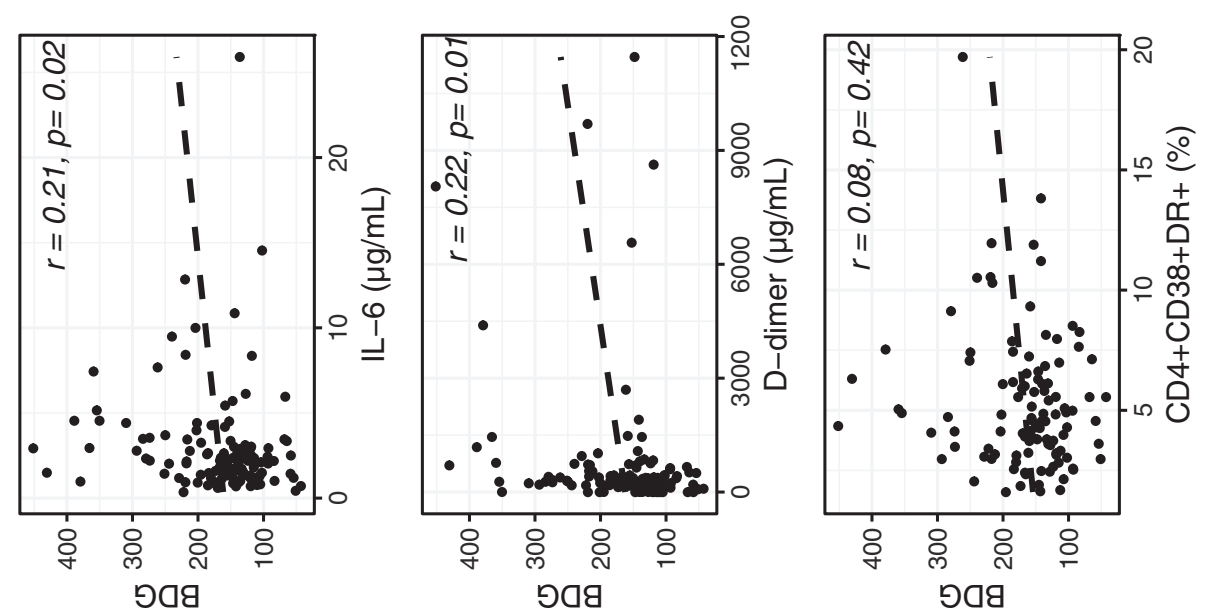

向

乌.

宅응

언 픙

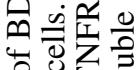

的家

造类.

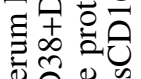

全㣢
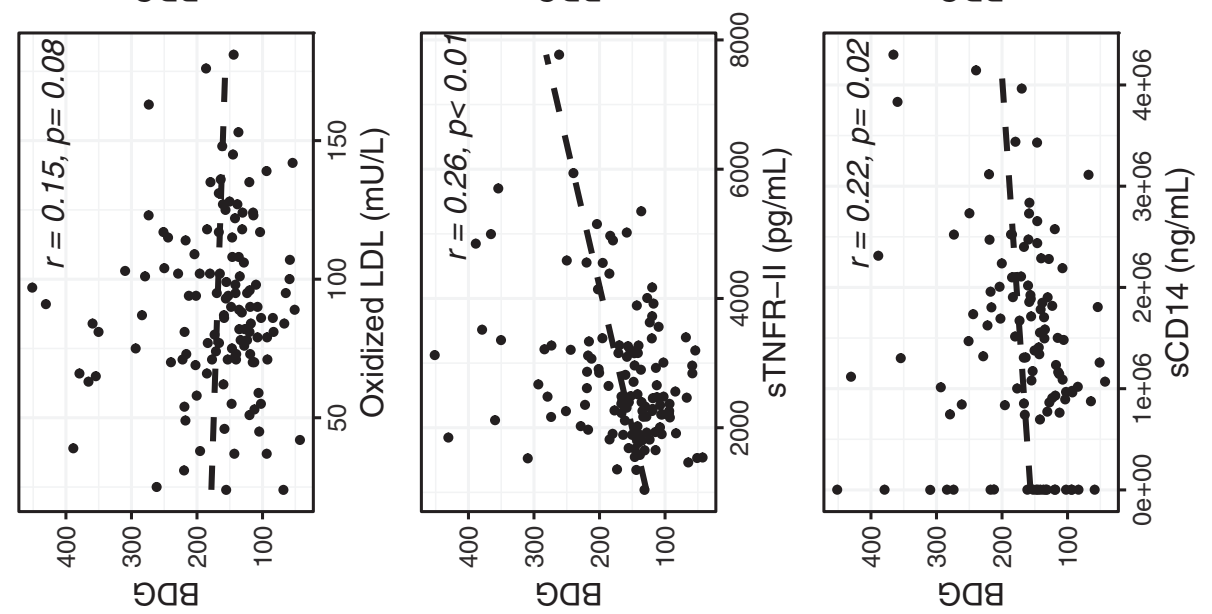



ठ U

。氞.

㐘造:

웡

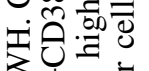

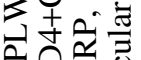

. 00

。ีษ

氖包.

드을

影焉
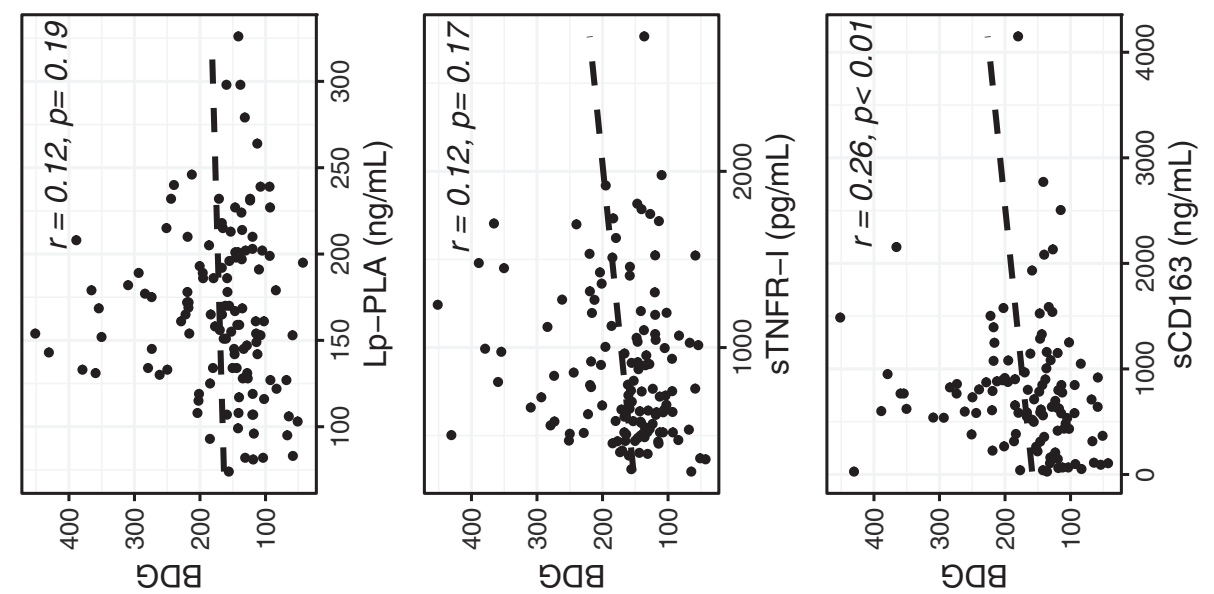

बiv

实当的

为茯

空导

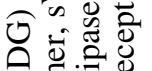

…․․ㅇ

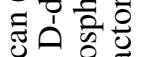

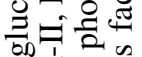

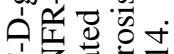

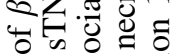

虽方令

这舀志

EZ

0
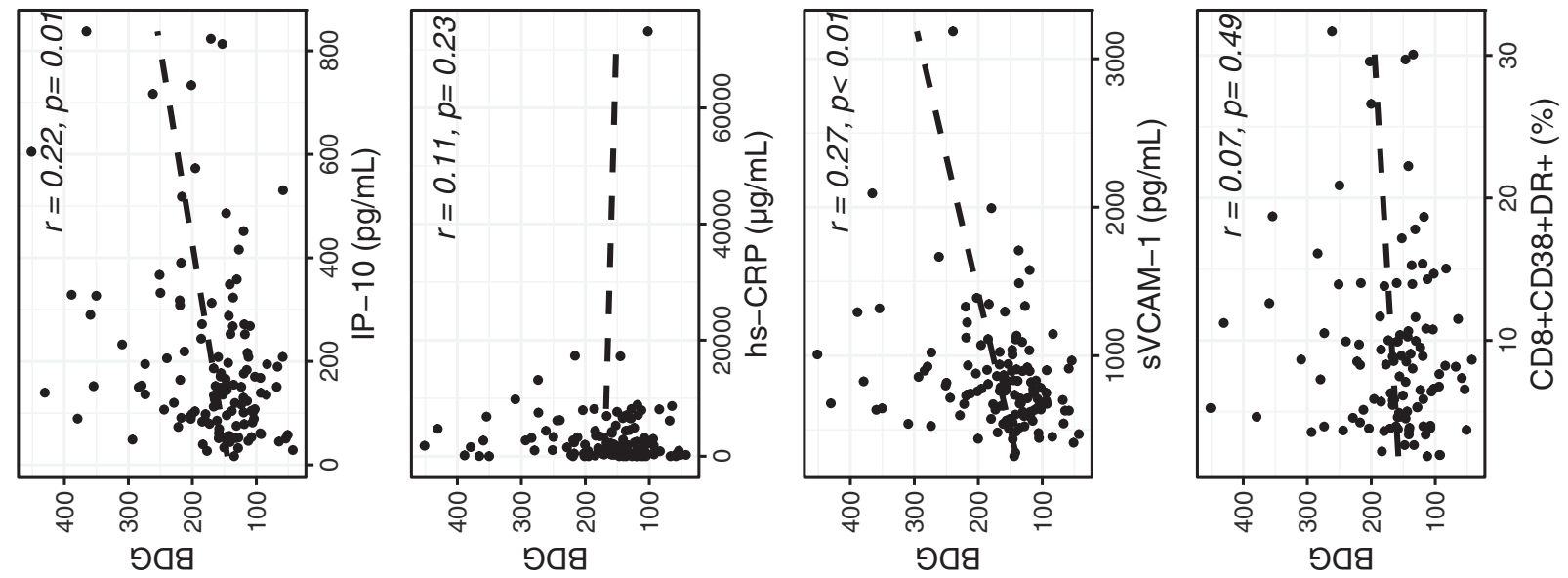

중유

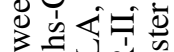

过定

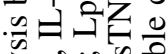

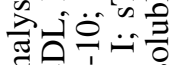

四毛的

웡

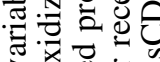

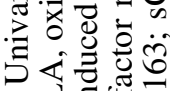

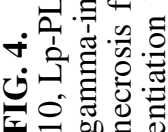



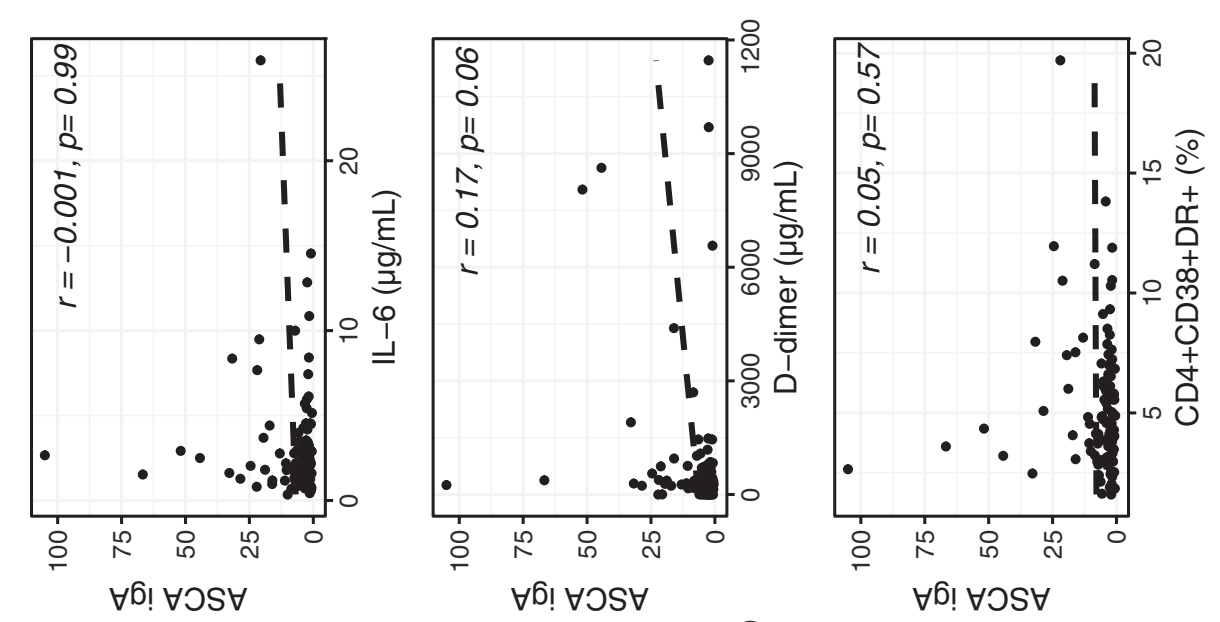

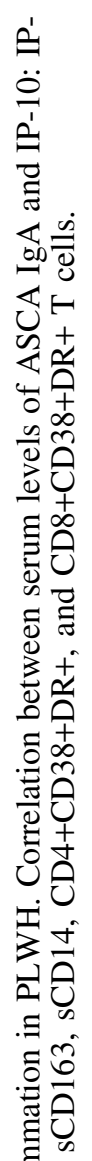
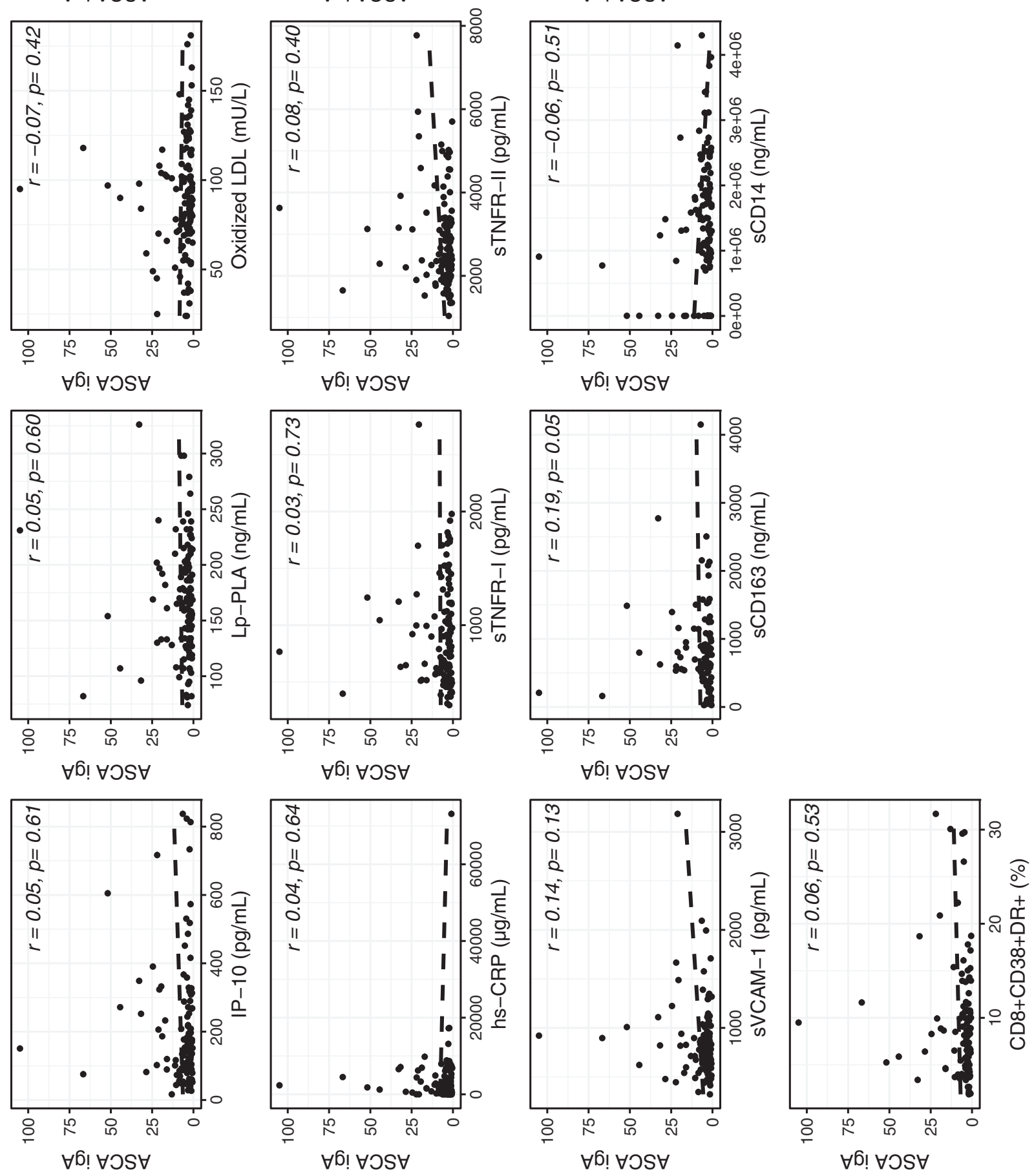

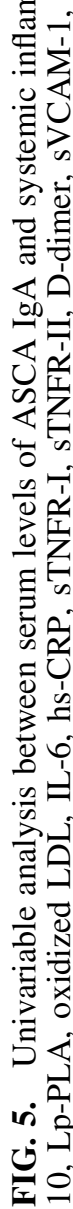



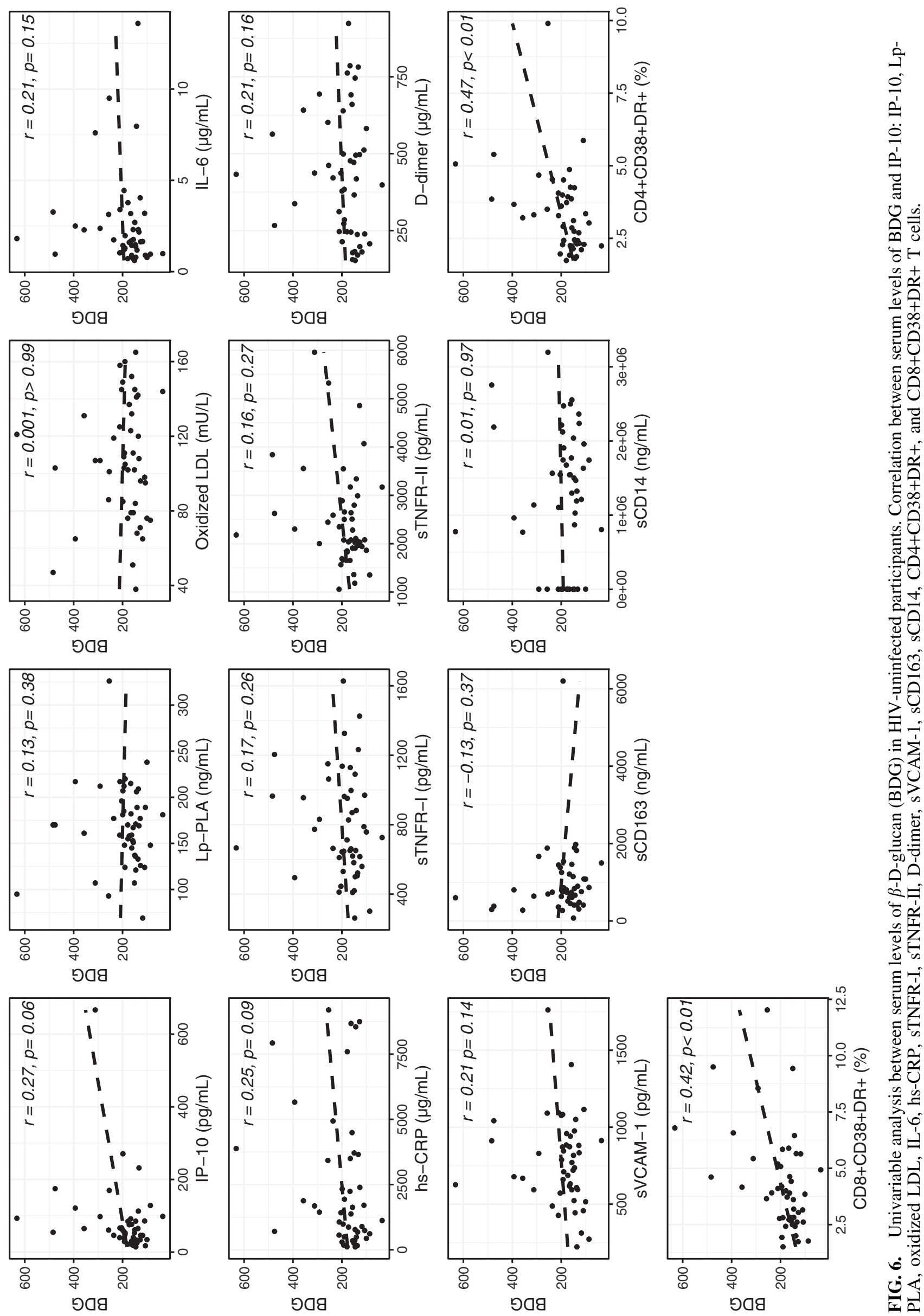

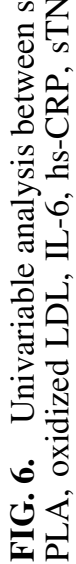



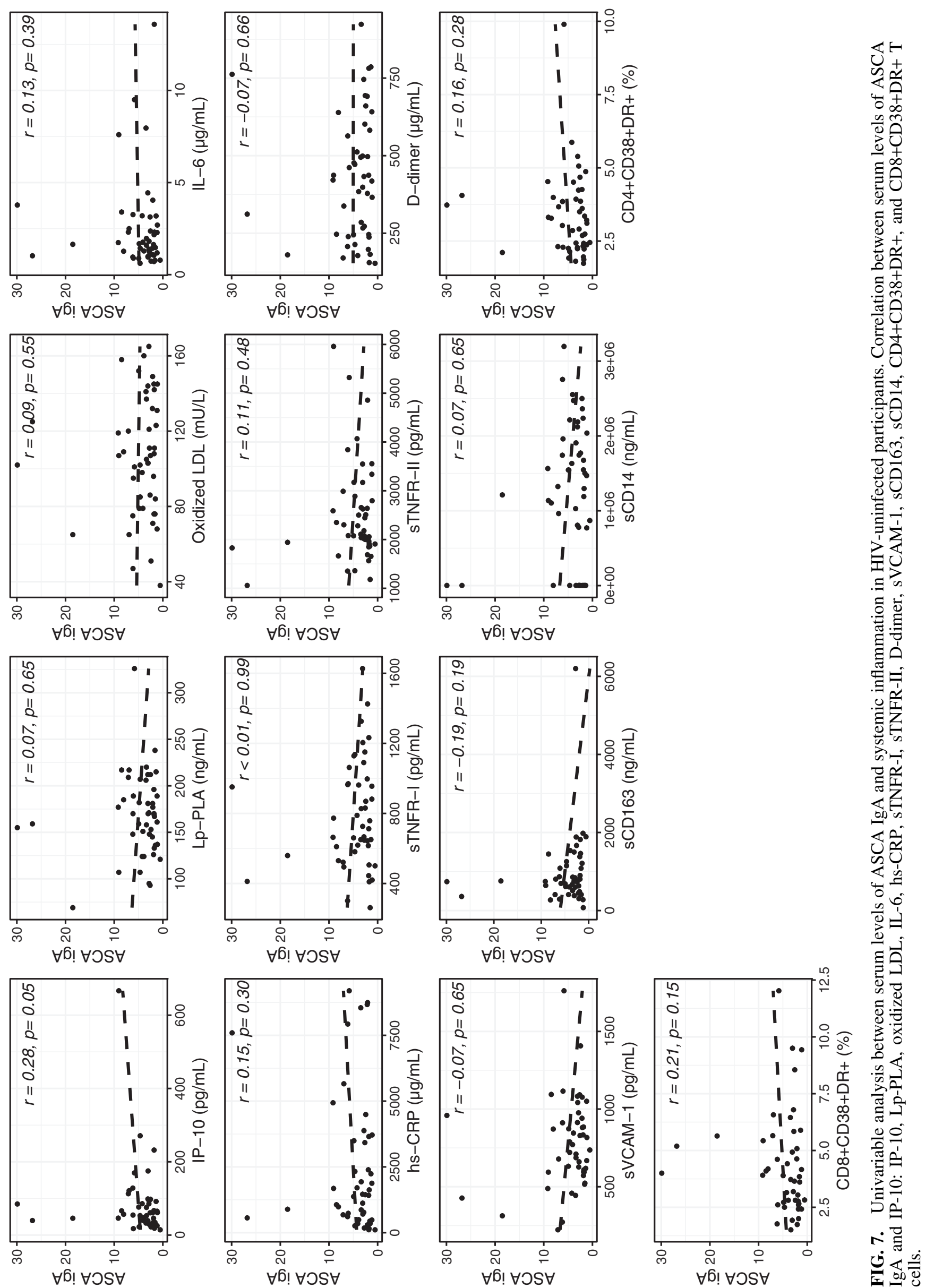
Table 2. Univariable Analysis Between Elevated Levels of Anti-SaCCHaRomyCES CEREVISIAE Antibodies IgA and Systemic Inflammation and HIV Variables (Spearman Correlation FACTORS WITH ASSOCIATED $P$ VALUES)

\begin{tabular}{|c|c|c|}
\hline & \multicolumn{2}{|c|}{ ASCA $\operatorname{IgA}(>3.08 \mathrm{U} / \mathrm{mL})$} \\
\hline & PLWH & HIV uninfected \\
\hline \multicolumn{3}{|l|}{ Inflammatory markers } \\
\hline IP-10 (pg/mL) & $-0.0012(p=.9922)$ & $0.213(p=.3175)$ \\
\hline Lp-PLA (ng/mL) & $-0.0314(p=.8024)$ & $-0.168(p=.4327)$ \\
\hline Oxidized LDL (mU/L) & $0.1274(p=.3041)$ & $0.1975(p=.3549)$ \\
\hline IL-6 $(\mu \mathrm{g} / \mathrm{mL})$ & $0.1184(p=.3326)$ & $-0.0165(p=.9389)$ \\
\hline hs-CRP $(\mu \mathrm{g} / \mathrm{mL})$ & $0.219(p=.0706)$ & $0.04(p=.8528)$ \\
\hline sTNFR-I (pg/mL) & $0.0053(p=.9658)$ & $-0.5235(p=.0087)$ \\
\hline sTNFR-II (pg/mL) & $0.0665(p=.5874)$ & $-0.2626(p=.2151)$ \\
\hline $\mathrm{D}$-dimer $(\mu \mathrm{g} / \mathrm{mL})$ & $0.2011(p=.0976)$ & $-0.1496(p=.4855)$ \\
\hline sVCAM-1 (pg/mL) & $0.0772(p=.5282)$ & $-0.1748(p=.414)$ \\
\hline sCD163 (ng/mL) & $0.1468(p=.263)$ & $-0.1296(p=.5462)$ \\
\hline $\mathrm{sCD} 14(\mathrm{ng} / \mathrm{mL})$ & $-0.3171(p=.012)$ & $-0.3696(p=.0755)$ \\
\hline $\mathrm{CD}^{+} \mathrm{CD} 38^{+} \mathrm{DR}^{+}(\%)$ & $-0.074(p=.5708)$ & $0.2302(p=.2906)$ \\
\hline $\mathrm{CD}^{+} \mathrm{CD}^{\circ} 8^{+} \mathrm{DR}^{+}(\%)$ & $0.0535(=.6821)$ & $0.4476(p=.0322)$ \\
\hline \multicolumn{3}{|l|}{ HIV variables } \\
\hline Viral load (copies/mL) & $-0.0051(p=.9666)$ & \\
\hline Absolute $\mathrm{CD}^{+}($cells $/ \mu \mathrm{L})$ & $-0.0622(p=.6172)$ & \\
\hline $\mathrm{CD}^{+}$count $($cells $/ \mu \mathrm{L})$ & $-0.1203(p=.3437)$ & \\
\hline Absolute CD4 nadir (cells $/ \mu \mathrm{L})$ & $0.0712(p=.5671)$ & \\
\hline ARV duration (months) & $0.0621(p=.6318)$ & \\
\hline CD4:CD8 ratio & $0.0745(p=.5588)$ & \\
\hline
\end{tabular}

Statistically significant values are bold.

$\mathrm{ARV}$, antiretroviral; IgA, immunoglobulin A; IL, interleukin.

\section{Fungal translocation and inflammatory markers}

BDG is known to be highly immunogenic. In HIV, Hoenigl et al. showed an association between BDG and sCD14, a marker of monocyte activation. ${ }^{30}$ Morris et al. demonstrated an association between higher levels of IL- 8 and TNF- $\alpha$ in PLWH and higher levels of circulating BDG. ${ }^{31}$ Our study yields novel insights into the potential roles played by fungal markers in HIV. We demonstrated a weak, but consistent, correlation between serum levels of BDG in PLWH and several markers of systemic inflammation, including sCD163, sCD14, IL-6, sTNFR-II, D-dimer, IP-10, and sVCAM-1, that was not seen in uninfected controls. This suggests that fungal translocation from an inflamed gut mucosa may contribute to the ongoing inflammation and immune activation seen in PLWH despite viral suppression on ART.

ASCA IgG and IgA did not show the same associations with inflammatory markers as BDG in PLWH. We hypothesized that this might be secondary to the inclusion of lower ASCA values, which may not necessarily reflect poor gut integrity. Therefore, a subgroup analysis was performed to evaluate if higher levels of ASCA IgA would have a stronger correlation with markers of systemic inflammation. This analysis did not yield any additional evidence to support that higher levels of ASCA IgA correlate with markers of systemic immune activation, highlighting the fact that ASCA may be a poor marker of intestinal mucosal integrity and fungal translocation in HIV.

\section{Study limitations}

Our study has several strengths, including the comprehensive evaluation of inflammation, and both innate and cellular arms of immune activation. Because this is a crosssectional analysis, we cannot prove causal relationships or exclude the possibility of residual confounding. Due to lack of prior data to guide sample size calculation, our study may not have been powered adequately to truly detect a difference between the groups and/or an association with measures of inflammation. The control group, despite being healthy and without any known active inflammatory conditions, had higher levels of cholesterol and D-dimer, which may have influenced our findings. Our population was also mostly men and African Americans, so our findings may not be applicable to other PLWH.

In addition, our study assumed that serum levels of BDG originated via GI translocation of candidal species, however, fungal organisms such as Histoplasma capsulatum have BDG as a surface moiety and candidal colonization involves organ systems beyond the GI tract. A limitation of our study is that we were not able to include measurements of the presence of other fungal species. We were also not able to investigate the microbiome of our participants nor measurements of serum LPS or other markers of gut integrity, such as zonulin, which may have been useful in demonstrating alterations in gut mucosal permeability.

\section{Conclusion}

In conclusion, our investigation provides preliminary and novel insight into the role of fungal translocation in persistent inflammation and immune activation in treated HIV. Although BDG was not altered by HIV-serostatus, its direct relationship with inflammatory markers in PLWH suggests a possible role of fungal translocation as a mediator of inflammation in PLWH. The role of the microbiome and fungal translocation in inflammation in PLWH warrants further investigation. 


\section{Acknowledgments}

The authors thank the patients who participated in this research. Funding: this work was supported by the National Institutes of Health [DK118757, HL126526, HL126539 to GAM], [R00HL108743 to NF], and [K23HD088295-01A1 to $\mathrm{SDF}$ ] and by an internal grant from University Hospitals Cleveland Medical Center.

\section{Authors' Contributions}

G.A.M. designed the study, obtained funding, and oversaw study evaluations and monitoring. A.S. and L.S. provided statistical support. N.F. assisted with the inflammation and immune activation marker assays. M.R., C.L.H., and M.A.G. assisted with the fungal translocation markers. L.W. assisted in the data analysis plan and wrote the first draft of the article. All authors contributed to data analysis and reviewed the article for intellectual content.

\section{Author Disclosure Statement}

G.A.M. served as a consultant for Gilead, GSK/Viiv, and Merck and has received research funding from Roche, Astellas, Gilead, Merck, and BMS. N.F. served as a consultant for Gilead. All other authors had no conflict of interest.

\section{References}

1. Marcus JL, Chao CR, Leyden WA, et al.: Narrowing the gap in life expectancy between HIV-infected and HIVuninfected individuals with access to care. J Acquir Immune Defic Syndr 2016;73:39-46.

2. Lewden C, Chene G, Morlat P, et al.: HIV-infected adults with CD4 count greater than 500 cells on long-term cART reach same mortality rates as general population. J Acquir Immune Defic Syndr 2007;46:72-77.

3. Phillips AN, Neaton J, Lundgren JD: The role of HIV in serious diseases other than AIDS. Aids 2008;22:24092418.

4. Neuhaus J, Jacobs DR, Jr., Baker JV, et al.: Markers of inflammation, coagulation, and renal function are elevated in adults with HIV infection. J Infect Dis 2010;201:17881795.

5. Burdo TH, Lentz MR, Autissier $\mathrm{P}$, et al.: Soluble CD163 made by monocyte/macrophages is a novel marker of HIV activity in early and chronic infection prior to and after anti-retroviral therapy. J Infect Dis 2011;204:154163.

6. Schouten J, Wit FW, Stolte IG, et al.: Cross-sectional comparison of the prevalence of age-associated comorbidities and their risk factors between HIV-infected and uninfected individuals: The AGEhIV cohort study. Clin Infect Dis 2014;59:1787-1797.

7. Longenecker CT, Sullivan C, Baker JV: Immune activation and cardiovascular disease in chronic HIV infection. Curr Opin HIV AIDS 2016;11:216-225.

8. Sainz T, Alvarez-Fuente M, Navarro ML, et al:: Subclinical atherosclerosis and markers of immune activation in HIVinfected children and adolescents: The CaroVIH study. J Acquir Immune Defic Syndr 2014;65:42-49.

9. Brenchley JM, Price DA, Schacker TW, et al:: Microbial translocation is a cause of systemic immune activation in chronic HIV infection. Nat Med 2006;12:1365-1371.
10. Marchetti G, Tincati C, Silvestri G: Microbial translocation in the pathogenesis of HIV infection and AIDS. Clin Microbiol Rev 2013;26:2-18.

11. Klatt NR, Funderburg NT, Brenchley JM: Microbial translocation, immune activation, and HIV disease. Trends Microbiol 2013;21:6-13.

12. Nazli A, Chan O, Dobson-Belaire WN, et al.: Exposure to HIV-1 directly impairs mucosal epithelial barrier integrity allowing microbial translocation. PLoS Pathog 2010;6: e1000852.

13. Caradonna L, Amati L, Magrone T, Pellegrino NM, Jirillo E, Caccavo D: Enteric bacteria, lipopolysaccharides and related cytokines in inflammatory bowel disease: Biological and clinical significance. J Endotoxin Res 2000;6:205-214.

14. Karageorgopoulos DE, Vouloumanou EK, Ntziora F, Michalopoulos A, Rafailidis PI, Falagas ME: Beta-D-glucan assay for the diagnosis of invasive fungal infections: A meta-analysis. Clin Infect Dis 2011;52:750-770.

15. Standaert-Vitse A, Jouault T, Vandewalle P, et al.: Candida albicans is an immunogen for anti-Saccharomyces cerevisiae antibody markers of Crohn's disease. Gastroenterology 2006;130:1764-1775.

16. Dambuza IM, Brown GD: C-type lectins in immunity: Recent developments. Curr Opin Immunol 2015;32:21-27.

17. Iliev ID, Funari VA, Taylor KD, et al: Interactions between commensal fungi and the C-type lectin receptor Dectin-1 influence colitis. Science 2012;336:1314-1317.

18. Funderburg NT, Jiang Y, Debanne SM, et al.: Rosuvastatin reduces vascular inflammation and $\mathrm{T}$-cell and monocyte activation in HIV-infected subjects on antiretroviral therapy. J Acquir Immune Defic Syndr 2015;68:396-404.

19. Matthews DR, Hosker JP, Rudenski AS, Naylor BA, Treacher DF, Turner RC: Homeostasis model assessment: Insulin resistance and beta-cell function from fasting plasma glucose and insulin concentrations in man. Diabetologia 1985;28:412-419.

20. Schietroma M, Carlei F, Cappelli S, Amicucci G: Intestinal permeability and systemic endotoxemia after laparotomic or laparoscopic cholecystectomy. Ann Surg 2006;243:359_ 363.

21. Jiang W, Lederman MM, Hunt P, et al.: Plasma levels of bacterial DNA correlate with immune activation and the magnitude of immune restoration in persons with antiretroviral-treated HIV infection. J Infect Dis 2009;199: 1177-1185.

22. Cassol E, Malfeld S, Mahasha P, et al.: Persistent microbial translocation and immune activation in HIV-1-infected South Africans receiving combination antiretroviral therapy. J Infect Dis 2010;202:723-733.

23. Funderburg NT, Zidar DA, Shive C, et al:: Shared monocyte subset phenotypes in HIV-1 infection and in uninfected subjects with acute coronary syndrome. Blood 2012; 120:4599-4608.

24. Cooke KR, Olkiewicz K, Erickson N, Ferrara JL: The role of endotoxin and the innate immune response in the pathophysiology of acute graft versus host disease. J Endotoxin Res 2002;8:441-448.

25. Underhill DM, Iliev ID: The mycobiota: Interactions between commensal fungi and the host immune system. Nat Rev Immunol 2014;14:405-416.

26. Chiba M, Mikami K, Iizuka M, et al.: Elevated plasma $(1 \rightarrow 3)$-beta-D-glucan, a fungal cell wall constituent, in a subgroup of Crohn disease. Scand J Gastroenterol 2001;36: 447-448. 
27. Guo Y, Zhou G, He C, Yang W, He Z, Liu Z: Serum levels of lipopolysaccharide and 1,3-beta-D-Glucan refer to the severity in patients with Crohn's disease. Mediat inflamm 2015;2015:843089.

28. van der Velden WJ, Netea MG, de Haan AF, Huls GA, Donnelly JP, Blijlevens NM: Role of the mycobiome in human acute graft-versus-host disease. Biol Blood Marrow Transplant 2013;19:329-332.

29. Kvehaugen AS, Aasbrenn M, Farup PG: AntiSaccharomyces cerevisiae antibodies (ASCA) are associated with body fat mass and systemic inflammation, but not with dietary yeast consumption: A cross-sectional study. BMC Obes 2017;4:28.

30. Hoenigl M, Pérez-Santiago J, Nakazawa M, et al.: $(1 \rightarrow 3)$ $\beta$-d-Glucan: A biomarker for microbial translocation in individuals with acute or early HIV infection? Front Immunol 2016;7:404.
31. Morris A, Hillenbrand M, Finkelman M, et al:: Serum $(1 \rightarrow 3)$-beta-D-glucan levels in HIV-infected individuals are associated with immunosuppression, inflammation, and cardiopulmonary function. J Acquir Immune Defic Syndr 2012;61:462-468.

Address correspondence to: Grace A. McComsey Division of Pediatric Infectious Diseases and Rheumatology University Hospitals Cleveland Medical Center 11100 Euclid Avenue Lakeside 1400 Cleveland, $\mathrm{OH} 44106$

E-mail: grace.mccomsey@uhhospitals.org 